The Be Phenomenon in Early-Type Stars, IAU Colloquium 175

ASP Conference Series, Vol. 214, 2000

M. A. Smith, H. F. Henrichs, and J. Fabregat, eds.

\title{
The Circumstellar Structure of the Be Shell Star $\phi$ Per
}

\author{
W. Hummel \\ Institut für Astronomie und Astrophysik und Universitätssternwarte \\ München, Scheinerstr 1, D-81673 München, Germany
}

S. Štefl

\begin{abstract}
Astronomical Institute, Academy of Sciences of the Czech Republic, CZ-25165 Ondřejov, Czech Republic
\end{abstract}

Th. Rivinius

\begin{abstract}
Landessternwarte Heidelberg, Königstuhl 12, D-69117 Heidelberg, Germany
\end{abstract}

\begin{abstract}
We present phase resolved spectra of the Be binary $\phi$ Per. It is shown that orbital phase variations of the He I line profiles are due to a sector emission region in the circumprimary disk facing the secondary. The increasing asymmetry of FeII emission lines indicates a density inhomogeneity in the circumprimary disk that developed after 1996.
\end{abstract}

\section{Introduction}

$\phi \operatorname{Per}(=\mathrm{HR} 496=\mathrm{HD} 10516)$ is a spectroscopic post case $\mathrm{C}$ mass transfer binary with a period of $P=126.67 \mathrm{~d}$ (Božic̀ et al. 1995) and a separation of $a=232 \mathrm{R}_{\odot}$ (Gies et al. 1998). The primary is a B0.5IVe star with $M_{V}=-3^{m} 11$. The outer $\mathrm{H} \alpha$ radius of its circumstellar disk was estimated to $63 \mathrm{R}_{\odot}$. The secondary is a sdO dwarf, with $T_{\text {eff }}=53000 \mathrm{~K}, \log g=4.2$.

Peculiar and complicated variability of the optical spectral lines indicate a more complex structure of the circumprimary disk than in single Be stars. The following spectral line components were detected (see also Poeckert 1981):

- Narrow HeI shell absorption profiles with maximum intensity at the superior conjunction.

- A broad double-peak HeII emission line varying in antiphase to primary absorption and circumstellar Fe II and Si II emission profiles.

- Weak narrow He I shell absorption appearing near quadratures and following RV of the secondary.

- Complex emission feature observed in some HeI lines and varying in antiphase with respect to the primary.

The aims of this study is to analyze orbital and long-term variations of $\mathrm{He} I$ and FeII emission lines. By their modeling we determine physical parameters of the region of their formation. For this purpose we use 67 spectra from the 

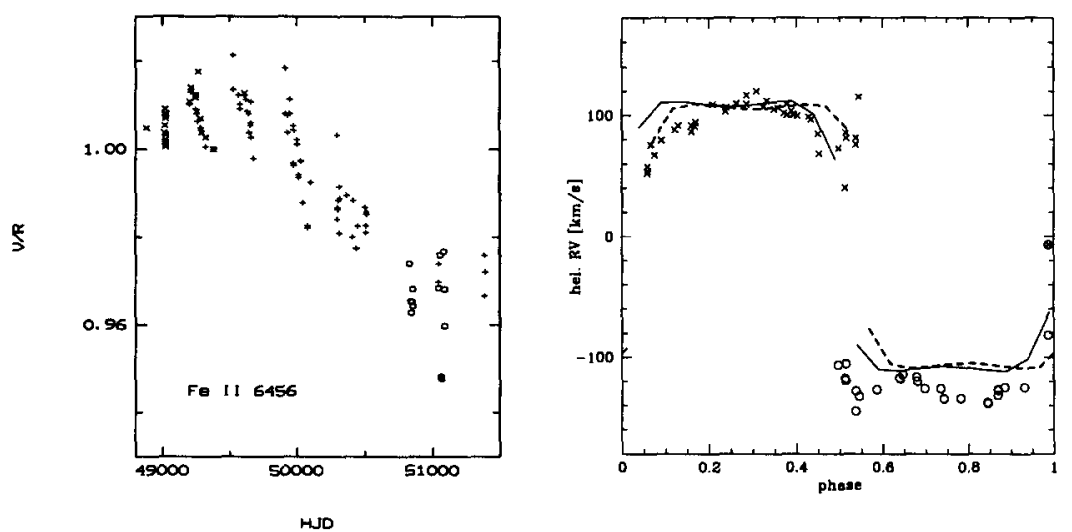

Figure 1. Left: Long-term V/R variations of FeII 6456: $\mathrm{x}-$ Božic et al. (1995), + - Ondřejov 2m, o - Heros 1998. Right: (box-diagram) Comparison between observed peak velocity of HeI 6678 (symbols) as a function of orbital phase and the same relation as predicted by the sector model (solid line) and the sphere model (dashed line).

Ondējov $2 \mathrm{~m}$ telescope obtained in 1993-1997 (6300-6700 $\AA$, linear dispersion $17 \AA / \mathrm{mm}), 16$ spectra obtained with the HERos spectrograph attached to the Heidelberg $0.72 \mathrm{~m}$ Waltz and $1.23 \mathrm{~m}$ Calar Alto telescopes in 1998 (3400 - 8600 $\AA$, resolving power 20000) and 21 high resolution profiles of $\mathrm{H} \alpha$, Fe II 5317 and He 15876 obtained at Calar Alto and Haute Province Observatory in 1993-1995 $(2.2 \mathrm{~m}$ and $1.52 \mathrm{~m}$ telescopes, respectively).

\section{Main results of observations}

After many years of symmetric Fe II emission profiles, documented also in our data till 1997, a strong asymmetry in the intensity of $V$ and $R$ emission components developed (see Fig. 1, left). The same trend can be detected also in the relative strength of maximum flux in the blue and red emission component of $\mathrm{He}$ lines. Such a parallel trend of HeI and FeII emission components indicates that they are all formed in the same circumprimary disk. The shape of asymmetric line profiles (after 1997) agrees well with profiles of single Be stars of class 2 (e.g. Hummel this volume). Consequently, we can interpret it as a growing density inhomogeneity, most probably a global disk oscillation, developing after 1996 . Orbital phase variations in connection with long-term variations offer an unique opportunity to study density perturbations in circumstellar disks of Be stars.

The main intention of using the Heros spectrograph was to search for the presence of emission component in individual He I lines in the optical region. Besides the known components in $\mathrm{He}$ I 6678 and 5876, we could identify similar components in HeI 7065, 5015 and 3889. All these lines correspond to $3^{i} \rightarrow 2^{j}$ 

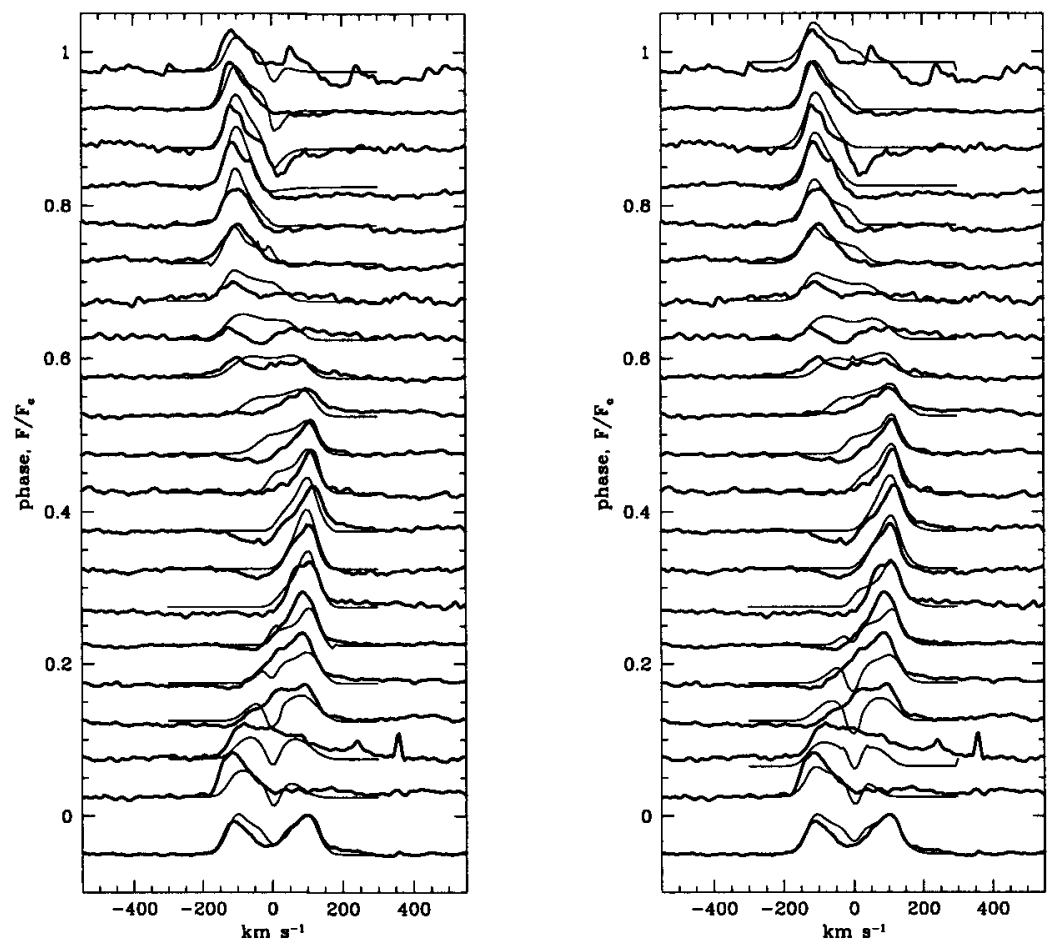

Figure 2. Bold profiles: Phase resolved observations of He I 6678 emission lines, corrected for phase shifted stellar absorption and phase binned. The lower most line profile is the orbital mean profile. Thin: phase dependent theoretical emission line profiles for He I 6678 from the sector model (left panel) and the sphere model (right panel)

atomic transitions, both in singlet and triplet lines. This selection effect may indicate a fluorescence emission mechanism but an exact modeling is needed to explain the emission.

Our monitoring enables detailed analysis of $\mathrm{He}$ I 6678 orbital variations. Both the dynamical phase spectra as well as measured emission intensities and radial velocities (Fig. 1, right) are not consistent with purely orbital velocity field.

\section{Line profile modeling}

The above results of observations lead to the hypothesis that the whole $\mathrm{He} I$ emission feature is formed in the outer part of the circumprimary disk illuminated by the secondary hot star. The physical consistency of such a model was verified by direct modeling of emission profiles. 
We assume one single circumprimary disk with an axi-symmetric density distribution in a binary potential with stellar parameters given by Gies et al. (1998) - inclination $i=80^{\circ}$, binary separation $\overline{R_{1} R_{2}}=29 R_{*}$ and $L_{1}$ at $19 \mathrm{R}_{*}$. The axi-symmetric column density distribution is described as $\Sigma \sim \Sigma_{0} R^{-0.5}$ and angular symmetric velocity field in binary potential as $V_{\text {rot }} \sim R^{-0.8}$. Emission lines are calculated as described by Hummel \& Vrancken (this volume).

$\mathrm{He}_{\mathrm{I}}$ is supposed to be excited in a region of the disk facing the secondary. The structure of this region is found by model fits in terms of a sector and in terms of a sphere around the secondary intersecting the circumprimary disk:

1. Sector model: emission region is constrained by the inner radius $R_{i}$ and outer radius $R_{d}$, azimuth sector $\Phi_{1}-\Phi_{2}$ and a phase delay $\Delta P$ with respect to the orbital phase

2. Sphere model: emission region is constrained by the outer radius $R_{d}$, radius of the parameterized excitation sphere around the secondary $R_{e}$ and the phase delay $\Delta P$.

By fitting the observed line profiles for each of the models we derived geometrical parameters $R_{i}=5, R_{d}=10, \Delta \Phi=112^{\circ}$ and $\Delta P=0.14$ for the sector model and $R_{d}=10, R_{e}=25.6$, and $\Delta P=0.16$ for the sphere model. The regions of the best fits for the both models coincide. They both explain well the orbital variations of the peak intensity and the box-shape of the peak velocity (Fig. 1, right) and match the orbital line profile variations of He I 6678 (Fig. 2).

\section{Conclusions}

The observations indicate that the orbital variation of the HeI 6678 feature is due to a partial photoionization in the circumprimary disk by the secondary $\mathrm{O}$ type star. The whole emission feature is formed in one disk and different origin of the two components is not required.

Modeling of both phase variations of He I emission lines and their scalar characteristics supports a model of a single axi-symmetric circumprimary disk with HeI excited only in the region facing the secondary. Consistent fitting of the line profiles provides the geometrical parameters of the disk and of its $\mathrm{He} I$ excited region. The disk radius is nearly $R_{\mathrm{d}}=10 R_{*}$, much smaller than the inner Lagrangian point $L_{1}$. The opening angle of the HeI emitting sector is $\Delta \phi \simeq 112^{\circ}$.

Acknowledgments. Financial support by the DFG and AV C̆R (436 TSE $113)$, and GA ČR $(202 / 97 / 0326)$ is gratefully acknowledged.

\section{References}

Božic̀, H., Harmanec, P., Horn, J. et al. 1995, A\&A 304, 235

Gies, D.R., Bagnuolo, W.G., Ferrara, E.C. et al. 1998, ApJ 493, 440

Poeckert, R., 1981, PASP 93, 297

Štefl, S., Hummel, W., Rivinius, Th., 1999 A\&A, submitted 